\title{
Improved Home Energy Management Techniques for Smart Grids
}

\author{
Wail Mardini", Firas Albalas, Qusai Obeidat and Muneer Bani Yassein \\ Computer science department, Jordan University of Science and Technology, \\ Irbid-Jordan \\ \{mardini,faalbalas,masadeh\}@just.edu.jo,qusai.gts@gmail.com
}

\begin{abstract}
Wireless Sensor Networks (WSNs) have become more integrated into our daily lives in various fields such as health, energy management systems, and safety applications. The use of WSNs in home environment applications is increasing rapidly. Smart homes implement Home Area Network (HAN) for many different applications including smart grids. in-Home Energy Management (iHEM) has been proposed in the literature to decrease energy expenses. In this paper, we propose to shift the consumers' demands to off-peak and mid-peak periods based on the devicelappliance priority. In our system we use three levels of appliance priorities; high priority, medium priority and low priority. We discuss using iHEM with different delays, and also augment iHEM with priority scheme and iHEM with different delays scheme to get better results. We simulate our work and compared it with the previous techniques using our discrete event simulator implemented in $\mathrm{C}++$. The main performance measure used to compare our work with the traditional iHEM scheme is the total savings in the cost of energy consumption in dollars. The results show that our proposed modifications over iHEM resulted in saving that ranges from around $15 \$$ to $30 \$$ during the six month period of the simulation.
\end{abstract}

Keywords: Smart Grids, Energy consumption, WSN, iHEM, Energy Management

\section{Introduction}

Global consumption of energy keeps increasing, and the demand for energy is expected to be doubled in 2020 [1]. The traditional power grids have been used for around a century and it suffers from several problems; including blackouts. The major reason of blackouts is the mismatch between demand and supply, and lack of monitoring tools and automation. Apparently, the existing power grid doesn't meet the requirements of the 21 st century. Smart grid technologies have been proposed in order to reduce the consumption of energy and solve many of the traditional grid problems when fully deployed [1].

In the smart grid, the consumer can produce local energy by using different energy generators, such as solar photovoltaic cell, wind and water power. The smart grid can provide the consumer with instant information including current rate and usage using smart meters. Many companies use Time-Of-Use (TOU) pricing that enables flexible billing. In TOU the price of electricity differs in peak, moderate and off-peak periods. During peak hours, consumers usually consume more energy, and thus the energy bill will increase as the rate is higher. Therefore, shifting consumers' demands, when possible, to off-peak hours will decrease their energy bill. Thus, it is a need to implement a smart management system to manage energy consumption in smart homes environments [1].

${ }^{*}$ Corresponding Author 
Smart grids are electricity networks that integrates all actions of all users connected to this network [2]. Such system allows for monitoring, analysis, control, improving efficiency, and reducing the energy consumption and cost. In smart grids, the consumer can produce local energy by using different energy generation methods such as solar photovoltaic cells, wind and water power. Figure 1 represents the concept figure of smart grid [3].

A smart grid can be also defined as a combination of hardware, reporting software and management that enables intelligent communication [4]. The consumer and companies in the world of smart grid have tools that enable users to manage and monitor energy consumption [4]. By using the smart grid technology, the electricity flows from the electricity company to consumers in a two-way fashion. The smart grids have many advantages for the consumers such as saving consumers' money by reducing the energy bill and allowing generating and sharing of local energy at consumers' homes or companies. A consumer can use energy from the storage unit and can sell the remaining one to the grid [5].

The communication network of a smart grid can be classified into three classes [6]:

1. Neighborhood Area Networks (NANs): A NAN is consisting of one or more base stations of third-generation $(3 \mathrm{G})$ and number of BANs.

2. Building Area Networks (BANs): A BAN is consisting of a number of apartments that having HANs.

3. Home Area Networks (HANs): Each HAN is a subsystem of the smart grid.

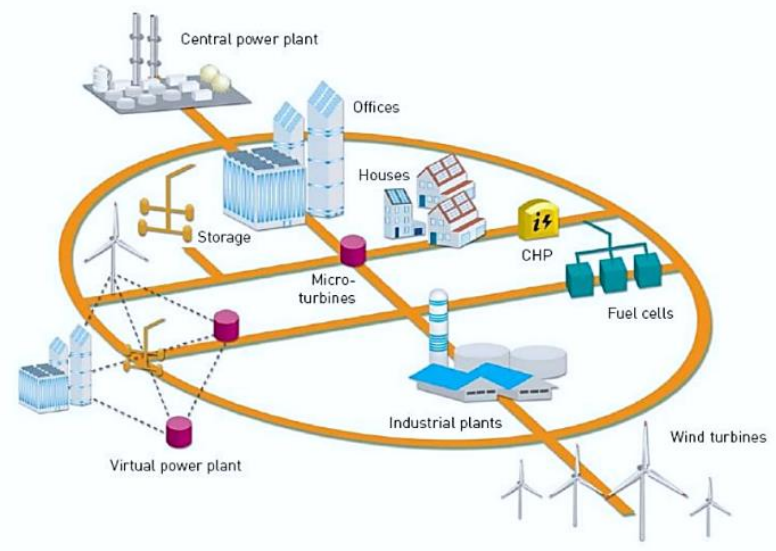

Figure 1. Smart Grid Concepts [3]

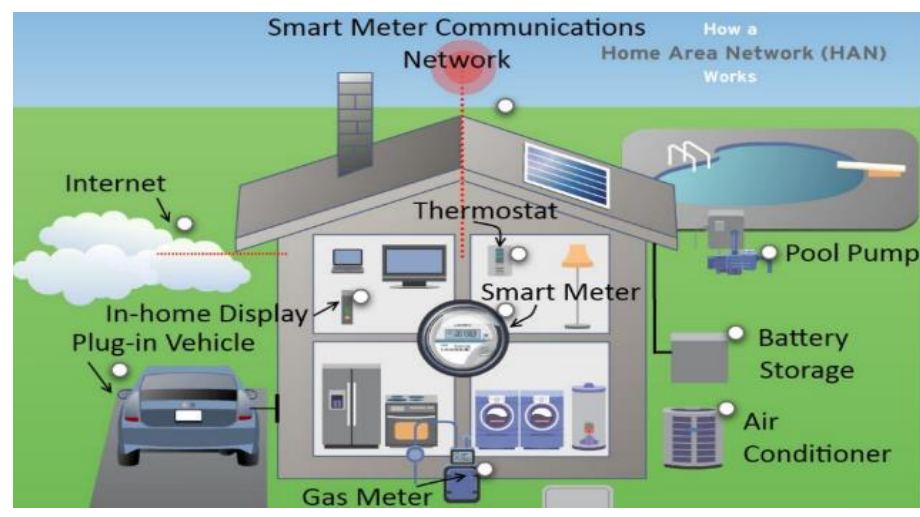

Figure 2. Home Area Networks [7] 
HAN is an important terminology in the smart grids. The smart grid application can communicate with several home appliances using HAN. The consumer can manage a smart home appliance using management systems according to the TimeOf-Use (TOU) pricing or other pricing techniques. The architecture of HANs is given in Figure 2 [7].

The paper consists of five sections. After the first section which include introduction about smart grids, section 2 discusses the literature review in the area of smart grids. Section 3 describes our methodology of the work and the scenarios description used in the testing phases. Section 4 presents and discusses our experiments and results. Section 5 contains the conclusions and suture works of the paper.

\section{Related Works}

In the literature, energy management systems have been studied in several works. There are many techniques that have been proposed and implemented to manage the consumption of energy and to reduce the energy bill. In this section, we will present some of the important papers that discuss different techniques of energy management systems in smart grids.

The same authors of [8] and [1] proposed an Appliance Coordination scheme (ACORD) and an improved version Appliance Coordination with Feed In (ACORD-FI) techniques in [9] and [10]. The aim of these two studies is to reduce the energy bill. In ACORD-FI scheme they use local energy generation, but on ACORD scheme they don't use local energy. In both algorithm when the resident turns on an appliance, a STARTREQ packet is sent to the EMU and in ACORD-FI sends to local energy to check if available energy on battery is enough to run the appliances, and then EMU shifts start time to off-peak period or start immediately. In these two schemes they simulate their work using discrete event simulation in $\mathrm{C}++$. They show that the result two of these schemes are reducing energy bills, they compare ACORD-FI with ACORD and NOACORD. They show that the result in ACORD-FI is better than ACORD schemes.

The authors in [11] proposed Smart Demand Responsive Energy Management System based on ZigBee/IEEE 802.15.4. The aim of this study is to save energy bill. The software design of the energy management system is presented in details, and they use different types of priority under a smart home energy management system concept. They use timeof-use (TOU) pricing on their scenario. Their system consists of sensor nodes and a PAN network coordinator. The sensor nodes collect data from home devices such as usage of energy and on-off switch. The proposed system applies on Thailand scenario using TOU. They study the customer profile that the usage will exceed $150 \mathrm{kWh} /$ Month. On the other hand, they also discuss the priority of load of home devices and they divide load prioritization into four parts: Uncontrollable loads devices that can't be controlled such as Lighting and Cooking Machines, Reparameterizable loads devices that is automatically controlled by temperature, such as Air Conditioners and Electric Water Heater, Interruptible loads devices that can be interrupted during working cycle such as Fans and Coffee Maker and Shiftable loads devices that can be shifted to another day period such as Hair Dryer and Clothes Washing Machines. The system they proposed works as follows; the unnecessary devices shifted to off-peak hours and the Interruptible loads part also shifted to off-peak hours. The test of this system is made on 30 household customers of Walailak University colleagues. By implementing this system they show the reduction of the energy bill and the system improves the usage of energy.

The authors in [12] proposed a Design and Implementation of User Interactive Wireless Smart Home Energy Management System. The architecture of this system is consisting of home appliances and each device contains a control device that is called Wireless Enabled Electricity Manager (WEEMAN). The control may be installed on 
every device or can have been on the switchboard; also, the system contains a smart meter. They use an algorithm that is called Availability Based Energy Management algorithm. The smart meter runs the algorithm; this algorithm will generate an efficient load pattern by using the previous usage data of appliances and by collecting the current consumption of appliances using WEEMAN. In this system the smart meter collects data from nodes (devices) and calculate total power, they use Time-Of-Use (TOU) pricing. The priority of devices is also mentioned in this paper. The home appliances are grouped in different categories: 1) Continuous, 2) Reserved, 3) User Priority, 4) and Delayable. The algorithm partition devices are based on device load, if the device operates of days in months more than $80 \%$, then the algorithm puts it in regular group and etc. They test their system for real devices, they connect Incandescent Bulb [Load A] and Fan [Load B], Load A means that the Incandescent Bulb is higher priority than Fan. When two devices are switched on the WEEMAN calculates the consumed power, then sends the data to the smart meter (controlling node). The smart meter runs the algorithm, and the algorithm will decide which device has higher priority than the other, the device with a high priority stays on, whereas the device with low priority will shift to switch on in the other period.

The authors in [13] proposed a Performance Evaluation of Energy Management System in Smart Home using Wireless Sensor Network. The aim of this system is to reduce energy cost. In this research paper. They implement their system using wireless sensor network and test it in a real environment. The users can manage their energy usage easier by using In-home display. The model they implemented is the first model of the three models they proposed; it consists of home appliances that are controlled by connected to the management system. The consumption of power and summary of performance is saved by the management system, then the total power consumption is measured by smart meter. The smart meter sends reports to the control center and the control center gives some suggestions of improvements based on the available information about the pattern of home power consumption. When suggestions are coming to the management system, some of them are applied, but the others are rejected. They test their system on the first model, because is simpler than other models and they test it in the laboratory of the department. The main factor that they work on is to cut off standby power, first, they measure appliance power consumption in the lab area; secondly, they measure power consumption in standby mode. By cutting off standby algorithm, the consuming power saved is $43 \%$, this is equal to saving energy cost to $57 \%$. Also, the system contains a GUI that is called In-Home Display (IHD), this IHD provides information to user about energy consumption and its cost.

The authors in [14] proposed a Home Appliances Coordination Scheme for Energy Management (HACS4EM) using Wireless Sensor Networks in Smart Grids. This scheme aims to reduce electricity bill of the consumer who uses this system in a way that shifts the operation of appliances from peak hours to off-peak hours. They organize the WSHAN in a cluster-tree topology, each appliance is represented as a RFDs and the smart meter is a FFDs. EMU is FFDs as Personal Area Network (PAN) coordinator. In their simulation, they used a scenario of four devices: washer, dryer, dishwasher and coffee maker, the energy consumption rate of all devices are: $0.89 \mathrm{kWh}, 2.46 \mathrm{kWh}, 1.19 \mathrm{kWh}$ and $0.4 \mathrm{kWh}$, respectively. They use TOU pricing scheme that has two peak hour period and one off-peak hour period. The simulation result shows that the peak load is reduced using this scheme as well as the electricity. Also, they evaluate the performance of WSHAN; they use 14 RFDs, four of them connected to appliances and five FFDs used for routing packets. The packet size they use in the application is between $32 \mathrm{~B}$ and $128 \mathrm{~B}$, and the interval is $10 \mathrm{~min}$. They show that the packet delivery ratio decrease when packet size increases and the delay increases when packet size increase.

The authors in [15] proposed an Efficient Approach for Home Energy Management System. This work aims to reduce energy expense; they evaluate the performance of iHEM with local energy generation, priority of appliances, and real-time pricing. In this 
scheme they use ZigBee for communication capability. The Wireless Sensor Home Area Network (WSHAN) is organized in a cluster-tree topology, the nodes in the living room are FFDs, the nodes in the bedroom and bathrooms are RFDs, smart meter is FFDs and EMU is a PAN coordinator. They use TOU pricing scheme with three periods; ONPEAK, MID-PEAK and OFF-PEAK. They use the same simulation parameter of [1] and the same devices. The results of this work show that the iHEM scheme reduces the energy bill almost 30\%, and the other result of the other performance evaluation is better when applying iHEM scheme.

The authors in [16] proposed a TOU-Aware Energy Management and Wireless Sensor Networks for Reducing Peak Load in Smart Grids. This paper aims to reduce energy bill and decrease usage of appliance during peak hours. In this energy management application, they use ZigBee IEEE 802.15.4 standard for communication between home appliances and management unit. This residential energy management scheme works as follows: when the consumer turn on the appliance, the START REQ packet sends to the energy manger (management unit), and then the energy manager computes the appropriate start time of the appliance. The decision made on the energy manager based on the local energy generation and the price of electricity. In their simulation, they analyze the use of four home appliances: dryer, coffee make, dishwasher and washer. They run the simulation for a period between 10 days and 210 days. The performance metrics they evaluate are the energy consumption cost, peak load ratio, and the experienced delay by the consumers. For peak load ratio, they show that when no energy management, the load almost 0.3 and when they employ the TOU-aware scheme, the loads of peak times almost 0.1 , this mean the residential energy management application reduce the peak load by $30 \%$.

The authors in [17] proposed a Smart Home Energy Management System for Monitoring and Scheduling of Home Appliances Using Zigbee. The proposed method of this paper consists of two main parts. The first part is the energy management center $(\mathrm{EMC})$ that containing a graphical user interface (GUI). The load scheduling is the second part of this method. They use LABVIEW to show the results of EMC and MATLAB simulations to show the results of load scheduling. Also, they implement a hardware model using human machine interface (HMI). This work aims to propose a model for Energy Management System (EMS). All information of the load consumption is contained in a text file. There are two appliances control provided in this system: using EMS interface and using the remote control. They used ZigBee protocol for communication between the appliances and to send data from appliances to EMS. This system is comfortable for consumers and is cost effective, and the consumer can control the home appliances easily by using remote or control. The GUI provides consumer with details of daily data consumption. They show the results when they use scheduler on load/Day KWH 15.80, the cost is 0.647 \$, and the cost of the same load of Non schedule is $0.75 \$$.

The authors in [18] proposed a Wireless Sensor Networks for Smart Grid Applications. The performance metrics of WSNs they evaluate are packet delivery ratio, delay and Packet Delay Variance (PDV). In this paper they used a ZigBee IEEE 802.15.4 communication protocol for data communication; ZigBee allows two types of devices: Reduced Functional Device (RFD) and Full Functional Device (FFD). They organize the WSN of their home model in a cluster-tree topology. They evaluate their performance metrics using Qualnet Network simulator, and they evaluate the performance of the network using different packet sizes and different number of nodes (network size). The performance metrics are packet delivery ratio, end-to-end delay and PDV. In network topology, there is one PAN coordinator and a number of nodes which are a mixture of RFD and FFD. They use interarrival time between 100s and 300s. They show that when the interarrival time increases, the packet delivery ratio and delay increases, but the PDV decreases when interarrival time increases. For varying network size (different number of 
nodes between 20 and 30), they show that when the network size is small, the packet delivery ratio is high that reaching $85 \%$ when the number of nodes is 20 . End-to-end delay and PDV increase when the number of nodes increases.

The authors of [19] presented details of various Home Energy Management Schemes (HEMs). This paper aims to increase savings on the energy bill, and to reduce Peak to Average Ratio (PAR) and peak demand. They present a various steps to have efficient energy consumption and they mention different pricing schemes such as Time Of Use (TOU), Critical Peak Pricing (CPP) and Real Time Pricing (RTP). The authors address two types of home energy management schemes, the first one is based on communications and the second one based on optimization. They present details of various techniques such as Optimization based residential energy management (OREM), in Home Energy Management Scheme (iHEM), ACORD, ACORD-FI and etc... OREM has an objective function to manage the consumption of energy by scheduling appliances at home in the convenient time slot. iHEM based on communication, they use Wireless Sensor Networks (WSNs) for appliances communication between them, the aim of iHEM is to shift the consumer load from on-peak period to off-peak or mid-peak to reduce energy bill and to reduce peak load demand. The aim of ACORD and ACORD-FI is the same of iHEM goal. This paper summarizes all these techniques to show the importance and the need of a home energy management system to get efficient energy consumption.

The authors of [20] proposed A Survey of Home Energy Management Systems in Future Smart Grid Communications. They present a review of various Home Energy Management System (HEMS) that aims to increase consumers saving, to reduce Peak to Average Ratio (PAR) and to deduce peak demand. In this survey they mention different pricing scheme such Time of Use (ToU) pricing and Real Time pricing (RTP). They also discuss a various techniques of energy management and make a comparison between them such iHEM, ACORD, ACORD-FI, Optimum Load Management (OLM) Strategy and Decision Support Tool (DST). iHEM, ACORD and ACORD-FI are discussed later on. The simulation results show that OLM reduce energy bill by $8-22 \%$. The goal of DST is to help the consumer to make an intelligent decision while the appliances are in running state. The results show that DST reduce energy bill by $16-25 \%$. This paper also presents some challenges of smart grids such as interoperability, scalability, Interdisciplinary and Security and Privacy.

The authors in [21] provided an extensive and comprehensive study on interactions and open issues of smart grids. In this paper they provide a comprehensive survey on a smart grid-driven approaches in energy efficiency information, communications infrastructures and data centers. Firstly, they provide a background of information of smart grids and then talk about approaches in energy-efficient communication system of smart grids. The energy-efficient communication system approaches of smart grids that mention are: Energy-Efficient Wireless Communication, Energy-Efficient Wire-line Communication and Energy-Efficient Optical Networks. Wireless communication is employed in a wide range application of smart grids, including, demand management, meter data collection and power line protection and monitoring. In wireless communication of smart grid section they provide a description of Smart Grid Wide Area Network (SG-WAN), Smart Grid Home Area Network (SG-HAN) and Smart Grid Neighborhood Area Network (SGNAN). In wire-line communication it can use SG-HAN and SG-NAN. In the optical networks may be used in SG-WAN and SG-NAN. At the end of this paper they provide a table that contains taxonomy of research that uses smart grid concepts in energy efficient Information and Communication Infrastructures (ICTs).

\section{Methodology}

The aim of this work is to decrease the total cost of energy consumption at home environment. In this section, we will first discuss the technique used in iHEM [15] with 
and without Feed-in, then we discuss our proposed changes and improvements to this technique.

\subsection{In Home Energy Management Scheme (iHEM)}

iHEM algorithm is proposed in [1]. The main parts of the algorithm could be grouped and described as follow. First, after all parameters were initialized, the main queue will be created as a linked list, and then initial events of all appliances are added to the main queue. Each event in the queue has two parameters; the first one is the type of event (start or stop) and the second is the timestamp of the event. This timestamp may start or stop time. The timestamp of events is computed assuming a Poisson Process Model. The interarrival time is a negative exponential distribution between two requests with random numbers of timestamps. Then, the algorithm check if the event start time is on peak hour, then try to shift it to off-peak or mid-peak depending on delay. The delay to the off peak period is calculated. If the delay to the off-peak is more than the maximum allowable delay (12 hour) [1], then the delay to the mid-peak is calculated. If the delay to mid-peak is more than maximum allowable delay (12 hour), the timestamp it remains as it is. Otherwise, the new time stamp will be generated from the first or the second case. If the start time of the event is in the mid-peak, then the delay to the off peak is calculated, if the delay is more than the maximum allowable delay ( 12 hour), the timestamp it remains as it is. If the device is not shifted because the delay is more than the maximum allowable delay, the device will start immediately.

The following process is repeated while the current time is less than simulation time. Choose the event with the smallest timestamp from the main queue.

- If the type of event is start event, then the process discussed in the previous paragraph is performed to either shift, or run the current event and schedule stop event in the main queue.

- If the type of event, stops event, then the energy consumption and the cost of energy consumption of the event is calculated according the device type.

The energy consumption is calculated according to (1) and the cost of the energy consumption is calculated according to (2).

\section{EnergyConsumption $=$ (Power * DurationTime) $/ 1000$}

where Power is the energy consumption of the appliances in watts and the Duration Time is the device working period in minutes.

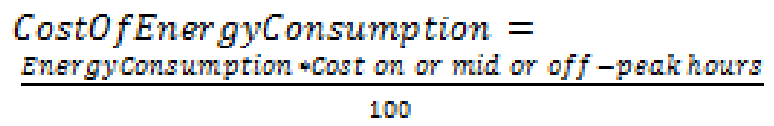

where the Energy Consumption is computed in equation (1) in kilowatt. The Cost on or mid or off-peak hours are the price of the period in cents; the period may be on peak hours, mid-peak hours, or off-peak hours.

In iHEM with Feed-in we assume the existence of PHEV battery storage at home. We assume that the model smart home has three Photovolatics (PV) panels that are able to generate $350 \mathrm{~W}$ per day. In this scheme, first the algorithm checks if the stored energy has enough energy that can run the appliance, then the device will starts immediately. This means that there is no change to the timestamp of the event. 


\subsection{Proposed Schemes}

\section{iHEM with Preemptive Priority Scheduling Scheme}

The aim of iHEM with Preemptive Priority is to further reduce the energy consumption and the total cost of energy bills. The concept of preemptive priority scheduling technique is applied to iHEM. The main goal is to try as much as possible to shift the event to mid or off peak hours for low priority devices and run a higher priority event in peak hours.

The difference between this technique and original iHEM is that the iHEM with preemptive priority is based on choosing the event with the next timestamp that has a high priority event when another event is in the running state. Traditional iHEM chooses the event with next timestamp only. In iHEM with the preemptive priority scheme, a high priority event will enter into the running state and a low priority event will enter the waiting state.

In iHEM with preemptive priority scheme, when the resident turns on an appliance, regardless peak hours (on-peak, mid-peak or off-peak), the appliance sends a start request (START-REQ) packet to the EMU. When the START-REQ packet receives by EMU, it communicates with the storage system battery to inquire about the available energy that is stored in the battery by sending an availablity request (AVAIL-REQ) packet to EMU. The prices of electricity are updated by a smart meter, whereas EMU communicates with smart meter to know about the updated prices. Available replay (AVAIL-REP) packet reply is sent from the storage unit to EMU that contains the information about the amount of stored energy of the battery. After that, EMU schedule appropriate starting time for the appliance by sending a start reply (START-REP) packet to notify the resident. Then, the resident sends a notification (NOTIFICATION) packet back to EMU with its decision.

If the device runs using storage battery, the EMU will send Update Available (UPDATE-AVAIL) packet to storage unit after receiving the user's decision to update the amount of energy in the storage unit. Figure 3 shows a message the flow for iHEM with Priority application.

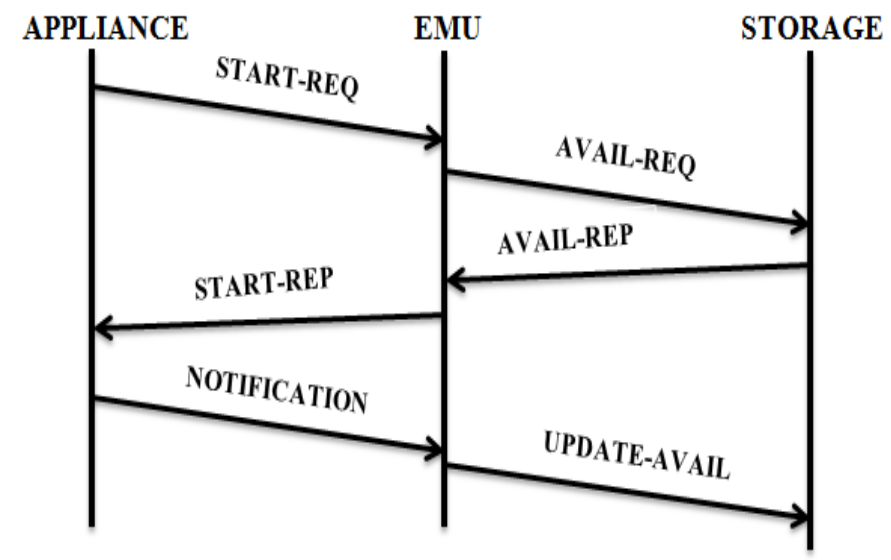

Figure 3. Message flow for iHEM with Priority

The algorithm of iHEM with preemptive priority is shown in Algorithm 1. The main queue, priority queue and waiting queue will be created as a linked list, and then we add events (appliances) to the main queue. Each event has four parameters. The timestamp of events is computed by a Poisson process Model, the inter-arrival time is a negative exponential distribution between two requests with random numbers of timestamp. 
For each event, the system check if the event start time is on peak hour, and then try to shift it to off-peak or mid-peak depending on the delay as in iHEM. During the continuous process we choose an event with the smallest timestamp from the main queue, priority queue and priority queue if it's not empty and assign each one to variable of type event. Compare between all events from three queues by the smallest timestamp and priority and choose one event to run it. We name the selected event as a current event. If the type of current event is start event, then check if there is any event arrived at the same time of current event running and check if it is priority is higher than current event priority where the priorities of all appliances (washer, dishwasher, dryer and coffeemaker) are 3,2, 4 and 1, respectively, where coffee maker has higher priority, if this is ok, then the highest priority event will stop the current event temporarily. The higher priority event will move to priority queue and current event (lower priority event) will move to waiting queue and we calculate the remaining time of waiting event and give it a new timestamp. If the timestamp is in peak, then we try to delay it to mid-peak or off-peak. If not, we will run the event and schedule stop event in the main queue instead of start event. If the type of event stops event, then we calculate energy consumption and the cost of energy consumption of the event which are computed in equation 1 and 2 according to the device turned on.

Algorithm 1: iHEM with Preemptive Priority Scheduling Scheme
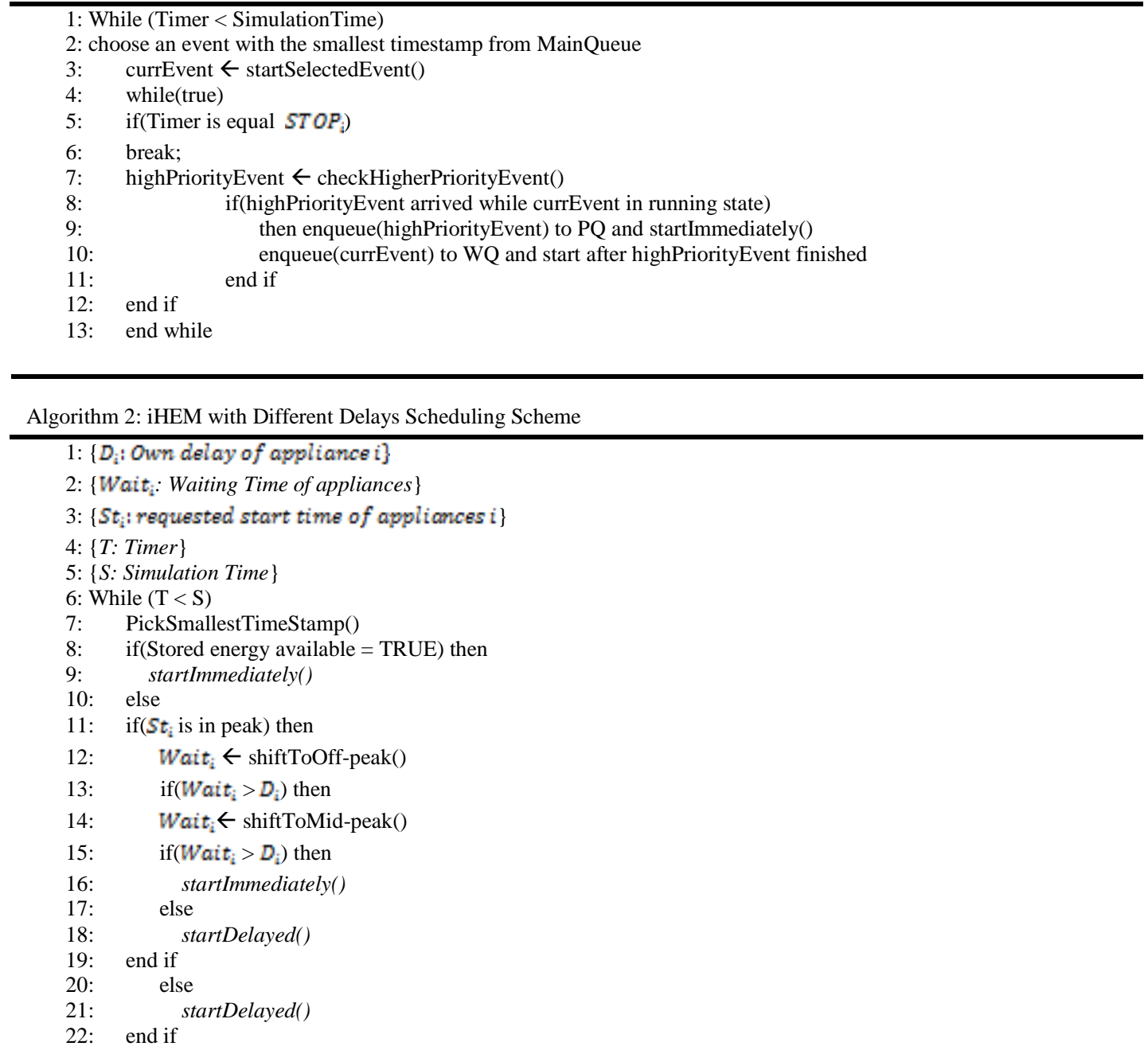


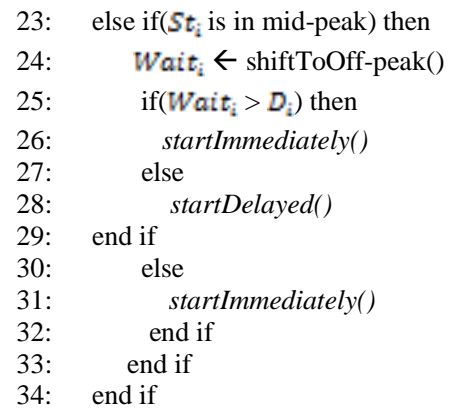

\section{iHEM with Different Delays Scheme}

The aim of this scheme is to reduce energy bill by shifting the maximum number of events to mid or off peak hours. The implementation of this scheme is the same of iHEM regular with one major difference in the allowable delay for each appliance. Each appliance has its own allowable delay such as: Washer: 15 hours and coffeemaker: 5 minutes. However, in traditional iHEM scheme we have a fixed delay of all appliances (12 hours) which is not convenient for consumers. Algorithm 2 shows how iHEM works with different delays work.

Each event has two parameters; the first one is the type of event (start or stop), the second is the timestamp of the event. The timestamp may start or stop time. The timestamp of events is computed by a Poisson process Model, the inter-arrival time is a negative exponential distribution between two requests with random numbers of timestamp which are computed in equation 1 and 2. The system checks if the event start time is on peak hour, and then try to shift it to off-peak or mid-peak depending on the delay. We calculate the delay to off peak. If a delay to off-peak is more than the maximum allowable delay of an appliances (based on appliance type: washer: 15 hours, dishwasher: 15 hours, dryer: 15 hours, coffeemaker: 5 minutes, AC: 0 hours and PHEV: 24 hours), then we calculate delay to mid-peak, if delay to mid-peak is more than maximum allowable delay (based on appliance type), the timestamp it remains as it is, else the new timestamp will be generated from the first case or second. If the start time is in mid-peak, then we calculate the delay to off peak. If the delay to off-peak is more than the maximum allowable delay (based on appliance type), the timestamp it remains as it is. If the device doesn't shift because the delay is more than the maximum allowable delay, the device will start immediately.

\subsection{Testing Scenarios}

The following is a description for all scenarios used in our experiments in order to evaluate the performance of all techniques discussed in section 3.1 and 3.2:

1) No energy management: this can be used as a reference case when no energy management is used at all.

2) iHEM regular (4 and 6 devices): this is the original algorithm of iHEM tested for cases of 4 and 6 basic devices. The main purpose of this scenario and the next one is to validate our implementation of iHEM technique. In this scenario, when the user request to run the device, the energy management unit checks if the request is in peak, and then calculate the delay to off peak. If the delay is more than maximum allowable delay (12 hour) then calculate delay to mid-peak, but if the delay is more than maximum allowable delay (12 hour) else, start immediately. If start time during midpeak, then calculate delay to off peak, but if the delay is more than maximum allowable delay (12 hour) else, start immediately. If the device doesn't shifts because 
the delay is more than the maximum allowable delay, the device will start immediately.

3) iHEM with feed in (4 and 6 devices): this is the same iHEM algorithm, but we use storage in home tested for cases of 4 and 6 basic devices. We assume that the model smart home has three PV panels that are able to generate $350 \mathrm{~W}$ per day. When the user requests to run the device, the energy management unit checks if the storage has enough energy to run the device and then start immediately, else check if the request is in peak and then calculate the delay to off peak, if delay is more than maximum allowable (12 hour), then calculate delay to mid-peak, if delay is more than maximum allowable (12 hour) else, start immediately. If start time is in mid-peak then calculate delay to off peak, if delay is more than maximum allowable (12 hour) else, start immediately. If the device doesn't shift because the delay is more than the maximum allowable delay, the device will start immediately.

4) iHEM with priority (4 and 6 devices): this is the basic iHEM algorithm integrated with preemptive priority. When the user requests to run the device the energy management unit checks if the request is in peak and then calculate the delay to off peak, if the delay is more than maximum allowable delay (12 hour), then calculate delay to mid-peak, if delay is more than maximum allowable delay (12 hour) else, start immediately. If start time during mid-peak, then we calculate the delay to off peak, if the delay is more than maximum allowable delay ( 12 hour) else, start immediately. If the device doesn't shift because the delay is more than the maximum allowable delay, the device will start immediately. The device with high priority will stop the device with least priority temporarily and delay it to another time. We calculate the remaining time of the delayed device, and we don't start it from the beginning.

5) iHEM with feed in and priority (4 and 6 devices): this is the same iHEM with feed in integrated with the use of preemptive priority. We assume that the model smart home has three PV panels that are able to generate $350 \mathrm{~W}$ per day. When the user requests to run the device, the energy management unit checks if the storage has enough energy to run the device and then start immediately, if the request is in peak, then calculate the delay to off peak. If the delay is more than maximum allowable delay (12 hour), then calculate delay to mid-peak, if the delay is more than maximum allowable delay (12 hour) else, start immediately. If start time is in mid-peak, then calculate delay to off peak, if the delay is more than maximum allowable delay (12 hour) else, start immediately. If the device doesn't shifts because the delay is more than the maximum allowable delay, the device will start immediately. The device with high priority will stop the device with least priority temporarily and delay it to another time. We calculate the remaining time of the delayed device, and we don't start it from the beginning.

6) iHEM with different delay 1 (4 and 6 devices): this is the iHEM regular algorithm implemented with different maximum allowable delay for the appliances and used for the washer 15 hours, dishwasher 15 hour, dryer 15 hours, coffeemaker 5 minutes, AC 0 hours and PHEV 24 hours. When the user requests to run the device the energy management unit checks if the request is in peak and then calculate the delay to off peak, if delay is more than maximum allowable delay (based of device type, then calculate delay to mid-peak, if delay is more than maximum allowable delay (based of device type) else start immediately. If start time is in mid-peak, then calculate delay to off peak, if the delay is more than maximum allowable delay (based on device type) else start immediately. If the device doesn't shifts because the delay is more than the maximum allowable delay, the device will start immediately.

7) iHEM with different delay 2 (4 and 6 devices): this is the iHEM regular algorithm implemented with different maximum allowable delay for the appliances and used for 
the washer 12 hours, dishwasher 12 hour, dryer 12 hours, coffeemaker 5 minutes, AC 0 hours and PHEV 24 hours. When the user requests to run the device the energy management unit checks if the request is in peak and then calculate the delay to off peak, if the delay is more than maximum allowable delay (based on device type then calculate delay to mid-peak, if delay is more than maximum allowable delay (based of device type) else, start immediately. If start time during mid-peak, then calculate delay to off peak, if the delay is more than the maximum allowable (based on device type) else, start immediately. If the device doesn't shift because the delay is more than the maximum allowable delay, the device will start immediately.

8) iHEM with priority and different delay ( 4 and 6 devices): this case represents the best of all proposed modifications; iHEM with priority algorithm and different maximum allowable delay. The maximum allowable delays used were: washer 12 hours, dishwasher 12 hour, dryer 12 hours, coffeemaker 5 minutes, AC 0 hours and PHEV 24 hours. When the user requests to run the device if the request is in peak and then calculate the delay to off peak. If delay is more than maximum allowable (based of device type), then calculate delay to mid-peak, if the delay is more than maximum allowable delay (based on device type) else, start immediately. If start time in midpeak then calculate delay to off peak, if the delay is more than the maximum allowable delay (based on device type) else, start immediately. If the device doesn't shift because the delay is more than the maximum allowable delay, the device will start immediately. The device with high priority will stop the device with least priority temporarily and delay it to another time, we calculate the remaining time of the delayed device, and we don't start it from the beginning.

9) iHEM with feed in and priority and different delay (4 and 6 devices): this case represents the best of all proposed modifications in addition to the use of feed-in PHEV; i.e. iHEM is used with priority algorithm and different maximum allowable delay. The maximum allowable delays used were: washer 15 hours, dishwasher 15 hour, dryer 15 hours, coffeemaker 5 minutes, AC 0 hours and PHEV 24 hours. We assume that the model smart home has three PV panels that are able to generate $350 \mathrm{~W}$ per day. When the user requests to run the device the energy management unit checks. If the storage has enough energy to run the device and then start immediately, else if the request is in peak and then calculate the delay to off peak, if the delay is more than the maximum allowable delay (based of device type), then calculate delay to mid-peak, if delay is more than maximum allowable delay (based of device type) else start immediately. If start time during mid-peak, then calculate delay to off peak, if the delay is more than the maximum allowable (based on device type) else start immediately. If the device doesn't shift because the delay is more than the maximum allowable delay the device will start immediately. The device with high priority will stop the device with least priority temporarily and delay it to another time, we calculate the remaining time of the delayed device, and we don't start it from the beginning.

\section{Experiments and Results}

In this section, we will discuss the different experiments and results to assess the performance of the proposed iHEM scheme and the proposed modifications that has been discussed in section 3. Our performance metric will be the total cost of energy consumption of home appliances. We measure it by simulating different scenarios as discussed in section 3.3. 


\subsection{Simulation Parameters and Assumptions}

We have different simulation parameters, some of them are applied in all scenarios and some parameters are applied in some scenarios. Table 1 presents the main parameters of the simulation which are the same used in [1].

Table 1. Simulation Parameters that are Used in All Scenarios

\begin{tabular}{|c|l|l|}
\hline & \multicolumn{1}{|c|}{ Parameter } & \multicolumn{1}{c|}{ Value } \\
\hline \hline 1 & Simulation-time & From 20 to 210 days (approximately 7 months) \\
\hline 2 & $\begin{array}{l}\text { Number } \\
\text { devices }\end{array}$ & 6 devices: Washer, Dishwasher, Dryer, Coffeemaker, AC and PHEV. \\
\hline 3 & Inter-arrival time & $\begin{array}{l}\text { Poisson process (during peak periods is a negative exponential distributed } \\
\text { with the mean of 12 hours while during off peak periods is negative } \\
\text { exponential distributed with mean of 2 hours) [1]. }\end{array}$ \\
\hline
\end{tabular}

Table 2 presents other parameters regarding energy consumption and average duration of the run time used in the simulations. All parameters have been used in all scenarios except the last column of priority, which has been used in some scenarios. We set the TOU rates in our simulation according to the prices mentioned in [1] as the prices used in the province of Ontario-Canada in 2011 (see Table 3).

Table 2. Simulation Parameters used in Some Scenarios

\begin{tabular}{|c|l|c|c|c|}
\hline & Appliance & Energy consumption (kWh) & Duration (min) & Priority \\
\hline \hline 1 & Washer & 0.89 & 30 & 4 \\
\hline 2 & Dishwasher & 1.19 & 90 & 3 \\
\hline 3 & Dryer & 2.46 & 60 & 5 \\
\hline 4 & Coffeemaker & 0.4 & 10 & 1 (highest priority) \\
\hline 5 & AC & 1.5 & 60 & 2 \\
\hline 6 & PHEV & 9.9 & 60 & 6 (lowest priority) \\
\hline
\end{tabular}

Table 3. TOU Rates in Ontario as of 2011 [1]

\begin{tabular}{|l|c|c|}
\hline TOU Period & Time & TOU Rate \\
\hline \hline On-Peak & $6: 00 \mathrm{am}$ to $12 \cdot 00 \mathrm{~nm}$ & $9.3 \mathrm{cent} / \mathrm{kWh}$ \\
\hline Mid-Peak & $12: 00 \mathrm{pm}$ to $6: 00 \mathrm{pm}$ & $8.0 \mathrm{cent} / \mathrm{kWh}$ \\
\hline On-Peak & $6: 00 \mathrm{pm}$ to $12: 00 \mathrm{am}$ & $9.3 \mathrm{cent} / \mathrm{kWh}$ \\
\hline Off-Peak & $12: 00 \mathrm{am}$ to $6: 00 \mathrm{am}$ & $4.4 \mathrm{cent} / \mathrm{kWh}$ \\
\hline
\end{tabular}

We simulate our schemes from 20 days to 210 days (approximately 7 months); the first 5 days are spared for warm up. We present results as an average of 15 simulation runs. Our simulation has been implemented as a discrete event simulation using $\mathrm{C}++$ under Ubuntu version 12.04.4 on HP Pavilion g6 core i5 with 4 GB RAM. The performance measure is to compare between different cases is the total costs in all approaches. 


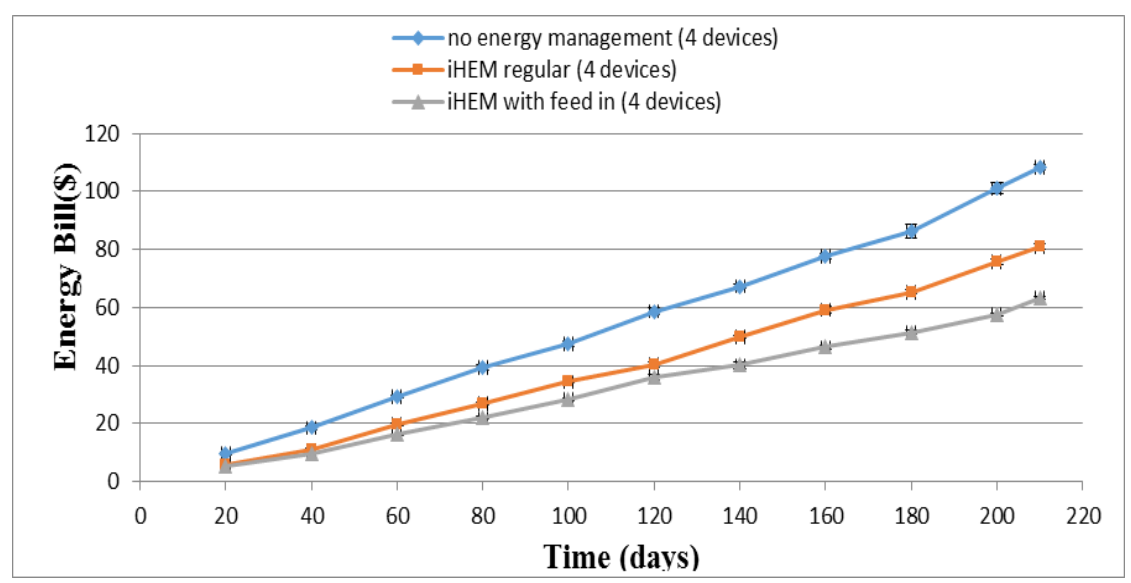

Figure 4. Total Cost for (No Energy Management), iHEM Regular and iHEM with Feed in (Local Energy Generation)

\subsection{Results and Discussions}

We first show some results to validate our implementation for the traditional iHEM, and then we show our work for the different scenarios discussed in section 3 compared to traditional iHEM.

Figure 4 shows the relation between Time (expressed in days) and the total cost of the consumed energy in dollar for the three scenarios; No energy management, iHEM and iHEM with feed in. The aim of these experiments is to verify our implementation of the traditional iHEM scheme discussed in [1] using the same parameters including the number of devices we obtained almost identical results as shown in that work. In this set of experiments we used four devices; washer, dishwasher, dryer and coffeemaker. The type of pricing used here is the same prices used in [1] which we had explained in table 1 . We run the simulation of these experiments from 20 to 210 days.

Figure 5 shows the relation between Time (expressed in days) and the total cost of the consumed energy in dollar for four scenarios; iHEM regular and iHEM with feed in. We have used four and six devices for two cases; the first and third scenarios are those similar to those discussed in Fig 4. In the second and fourth scenario, we simulate iHEM regular and iHEM with feed in by using six devices. We run the simulation of these experiments from 20 to 210 days. The total cost of iHEM regular (4 devices) is $\$ 80$ and $\$ 136$ of iHEM regular (6 devices) for 210 days; however the total cost of iHEM with feed in (4 devices) is \$ 63 and \$ 109 of iHEM with feed in (6 devices) for the same period, which gains a saving of approximately $\$ 17$ with four devices and 25 with six devices.

Figure 6 shows the relation between Time (expressed in days) and the total cost of the consumed energy in dollar for different scenarios; iHEM without priority (regular) and iHEM with priority. We have used four devices and six devices. The first and third scenarios are those similar to those discussed in Fig 4. The second and fourth scenarios are iHEM with preemptive priority using four and six devices, which as can be seen, that introduces some improvement. iHEM with priority introduce preemptive priority of the use of devices as explained in section 3 . The total cost of iHEM with priority (4 devices) is $\$ 65$ and $\$ 109$ by using (6 devices) for 210 days; however, the total cost of iHEM without priority (4 devices) is $\$ 80$ and $\$ 136$ by using (6 devices) for the same period, which gains a saving of approximately $\$ 15$ with four devices and 25 with six devices.

Figure 7 shows the relation between Time (expressed in days) and the total cost of the consumed energy in dollar for different scenarios; iHEM with feed in without 
priority (regular) and iHEM with feed in and with priority. We have used four devices and six devices. The first and third scenarios are those which are similar to those discussed in Fig 4. The second and fourth scenario is iHEM with feed in with preemptive priority using four and six devices which, as can be seen, that introduces some improvement. iHEM with feed in with priority introduce preemptive priority of the use of devices as explained in section 3. The total cost of iHEM with feed in and priority (4 devices) is $\$ 52$ and $\$ 91$ by using (6 devices) for 210 days; however, the total cost of iHEM without priority (4 devices) is $\$ 63$ and $\$ 109$ by using (6 devices) for the same period, which gains a saving of approximately $\$ 11$ with four devices and 18 with six devices.

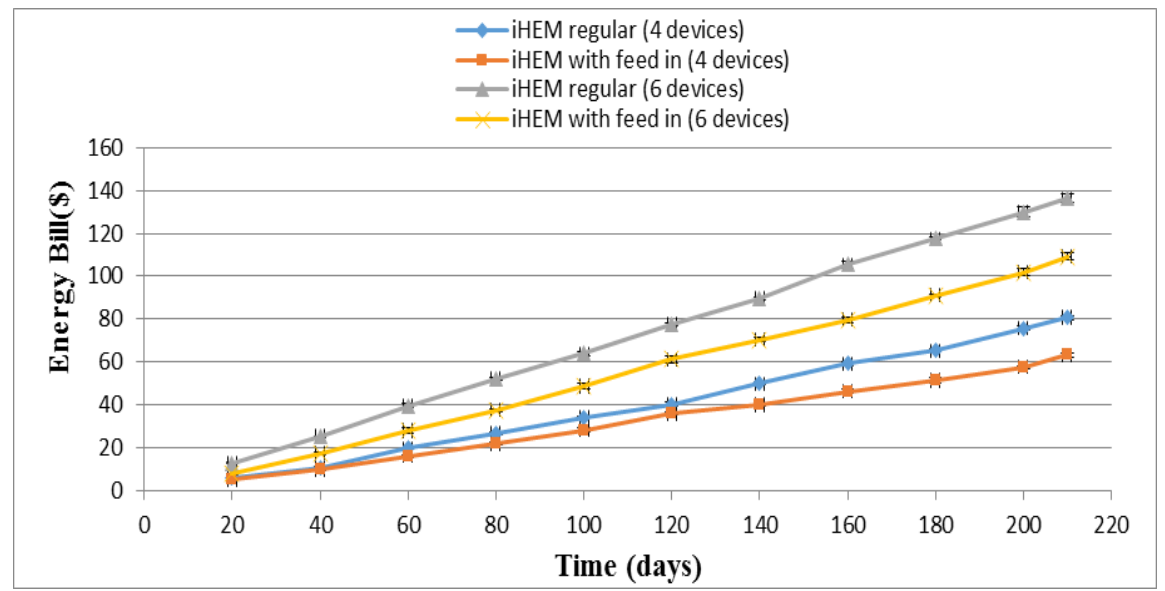

Figure 5. Total Cost for iHEM Regular and iHEM with Feed in (local energy generation) using Four and Six Devices

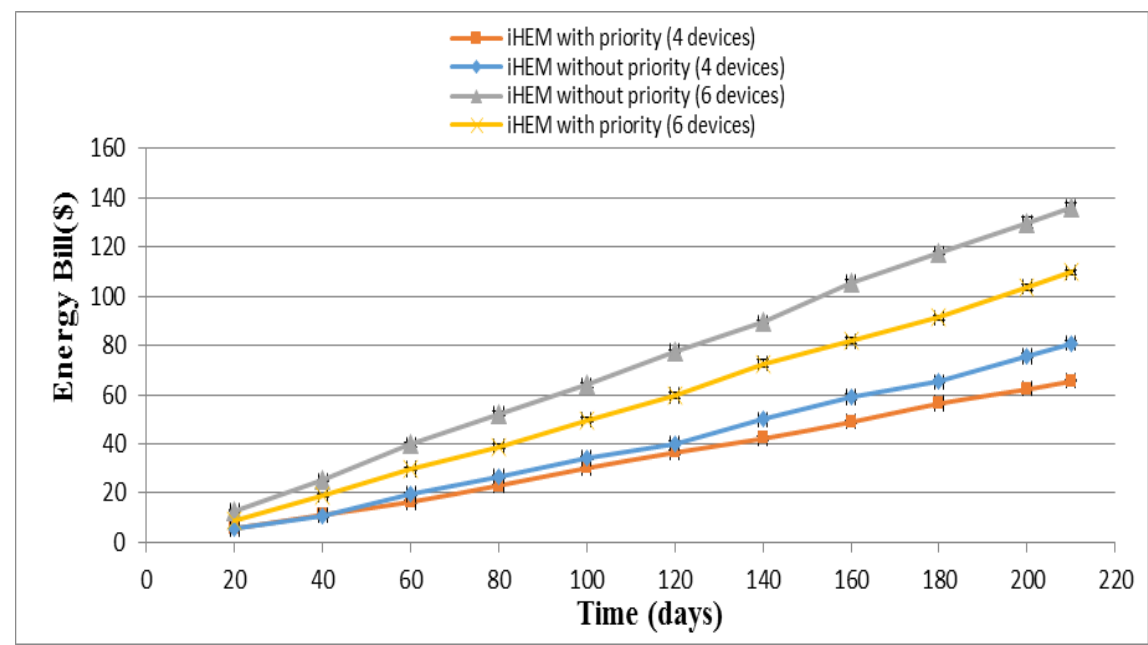

Figure 6. Total Cost for iHEM with and without Preemptive Priority using Four and Six Devices

Figure 8 shows the relation between Time (expressed in days) and the total cost consumed energy in dollar for three scenarios: iHEM with the fixed delay and iHEM with different delay, We have used four and six devices for all cases. In iHEM with the fixed delay scenario we use a fixed value as a maximum allowable delay of the appliance when we try to shift it from period to another and we use different value as a maximum allowable delay for iHEM with different delay. When we change the maximum delay of some appliances, we will get improvements of saving money. The total cost of iHEM with different delay with four devices is \$ 67 and \$ 113 with 
six devices for 210 days; however, the total cost of iHEM with fixed delay with four devices is $\$ 80$ and $\$ 136$ with six devices for the same period, which gains a saving of approximately $\$ 13$ with four devices and \$23 with six devices.

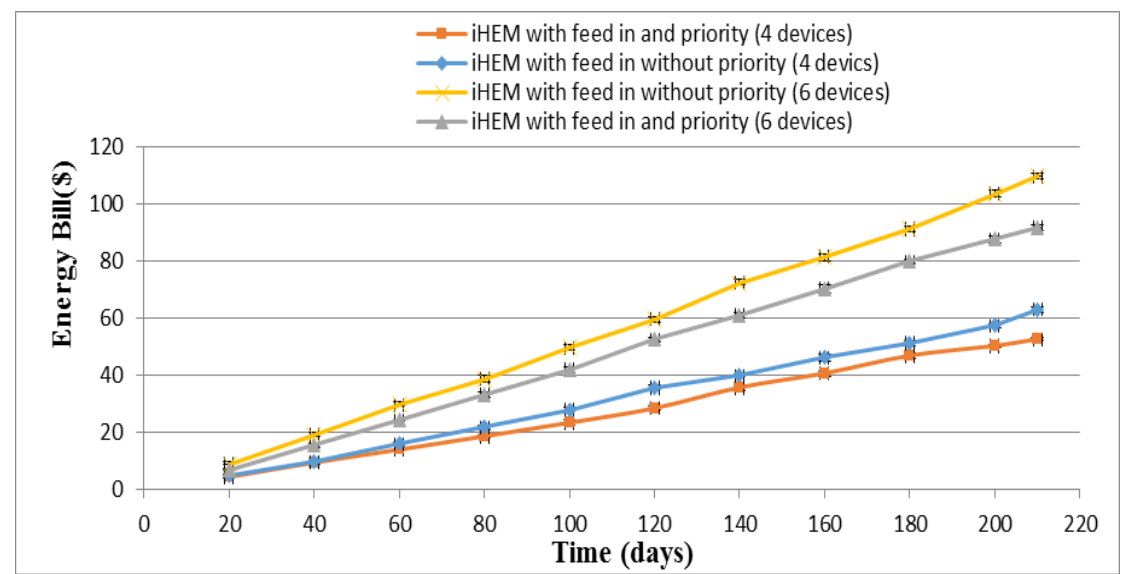

Figure 7. Total Cost for iHEM with Feed in (with and without Preemptive Priority) using Four and Six Devices

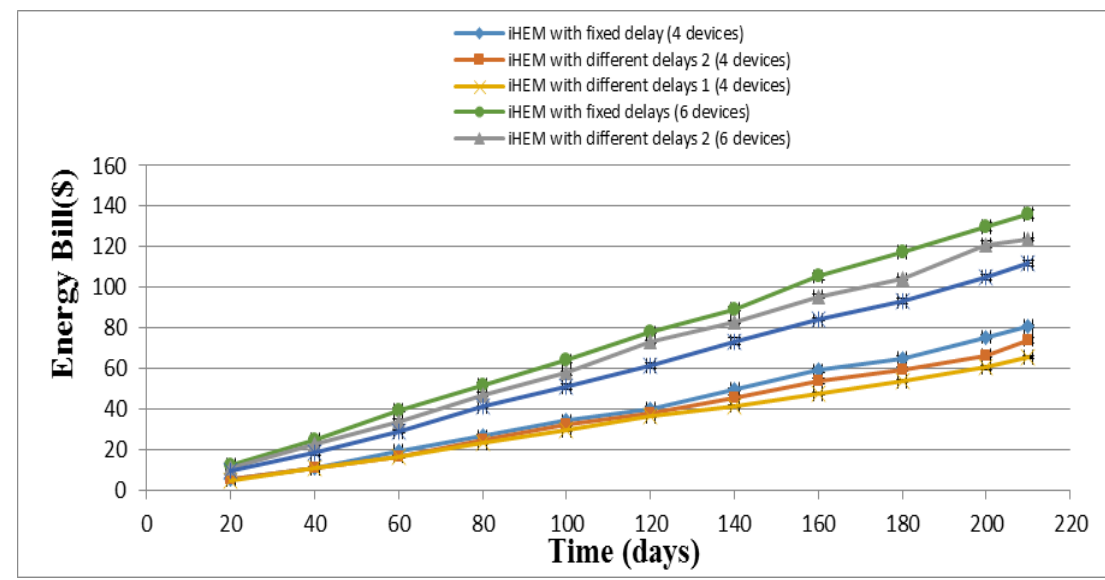

Figure 8. Total Cost for iHEM with Fixed Delay and Different Delays by using Four and Six Devices

Figure 9 shows the relation between Time (expressed in days) and the total cost of the consumed energy in dollar for different scenarios. iHEM regular, iHEM with priority and iHEM with priority and different delay. We have used four devices and six devices. We show that when we use different delay of appliances with priority, we will reduce energy bill more. The total cost of iHEM priority and different delay (4 devices) is $\$ 53$ and $\$ 95$ by using (6 devices) for 210 days; however, the total cost of iHEM (4 devices) is $\$ 80$ and $\$ 136$ by using ( 6 devices) for the same period, which gains a saving of approximately \$27 with four devices and \$21 with six devices.

Figure 10 shows the relation between Time (expressed in days) and the total cost of the consumed energy in dollar for different scenarios; iHEM with feed in, iHEM with feed in and priority, and iHEM with feed in and priority and different delay. We have used four devices and six devices. We show that when we used local energy generation with different delay of appliances with priority, we will reduce energy bill more. The total cost of iHEM with feed in priority and different delay (4 devices) is $\$ 43$ and $\$ 81$ by using (6 devices) for 210 days; however, the total cost 
of iHEM (4 devices) is $\$ 63$ and $\$ 109$ by using ( 6 devices) for the same period, which gains a saving of approximately $\$ 20$ with four devices and \$28 with six devices.

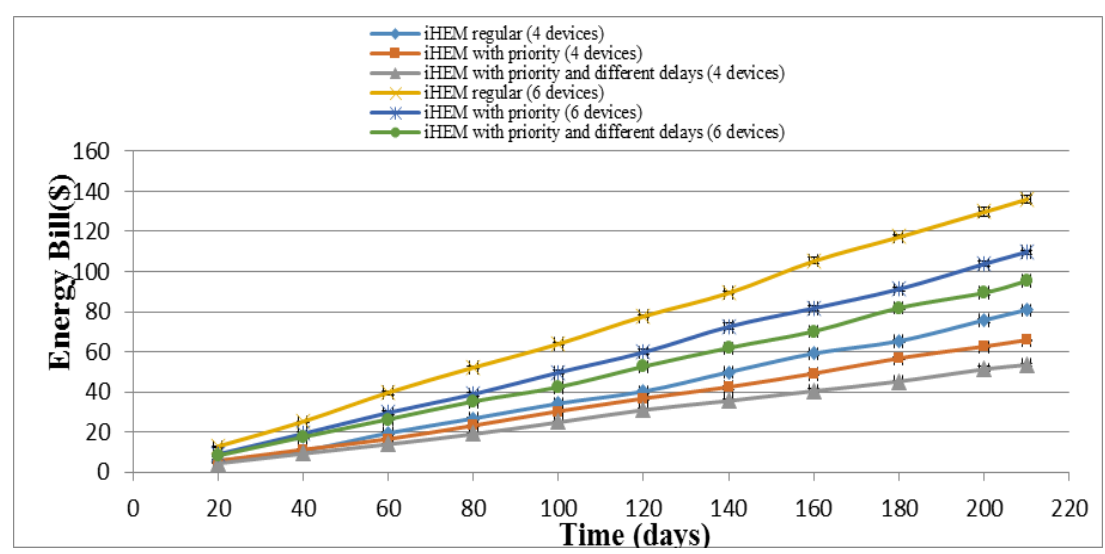

Figure 9. Total Cost for iHEM (without feed in) with Priority and Variant Delay by using Four and Six Devices

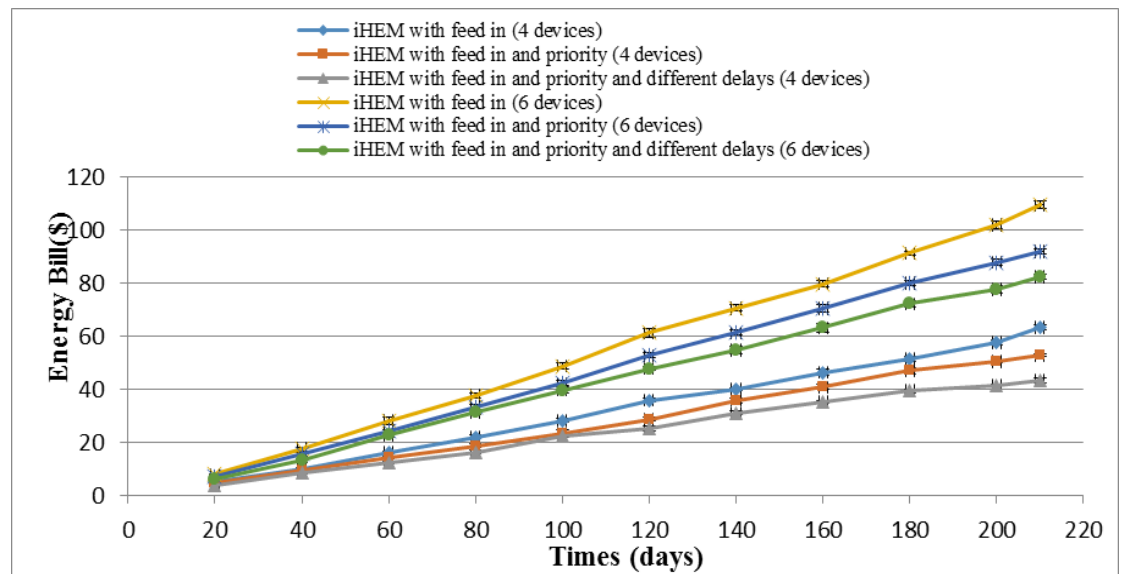

Figure 10. Total Cost for iHEM with Feed in and Priority and Variant Delays by using Four and Six Devices

\section{Conclusions and Future Works}

In our approach we aimed to shift the consumers' demands to off-peak and mid-peak periods based on the device/appliance priority. In our system we use three levels of appliance priorities; high priority, medium priority and low priority. We discuss using iHEM with different delays, and also augment iHEM with priority scheme and iHEM with different delays scheme to get better results

Many experiments have been conducted to study the behavior of our proposed schemes in this work. Our proposed schemes, including: iHEM with a preemptive priority scheduling scheme and iHEM with different delays scheduling scheme. The main goal of these experiments and works is to reduce total cost of energy bills, reduce total energy consumption on the home environment and reduce peak loads as much as possible. To obtain this goal we are trying to shift peak loads off-peak and mid-peak hours. The results show that iHEM with priority scheme is better than traditional iHEM saving of the cost; also iHEM with different delays saving of the cost is better than traditional iHEM. When we integrate iHEM with priority and iHEM with different delays together we are obtaining the highest savings of the cost regardless on the number of devices. 
Our work improved the energy cost regarding to iHEM scheme by adding new scheduling algorithms, in our future work we will try to investigate a new scheduling algorithms to be compared with our work. Also, we are planning to include learning techniques from the Artificial Intelligence (AI) field to increase consumer comfort and pervasiveness of our application. Furthermore, our schemes can be extended to a new class of appliances that allow subcycle scheduling. The availability of such appliances will enrich the opportunities of residential demand management applications.

\section{Acknowledgment}

The authors of this paper would like to thank Dr. Yaser Khamayseh for his help in the implementation of the codes in this paper.

\section{References}

[1] Erol-Kantarci M, Mouftah HT. Wireless Sensor Networks for Cost-Efficient Residential Energy Management in the Smart Grid. IEEE Transactions on Smart Grid on. June 2011; 314-325.

[2] Expert Group 1. Functionalities of smart grids and smart meters. EU Commission Task Force for Smart Grids, December 2010.

[3] Janez Potoc nik. European SmartGrids Technology Platform: Vision and Strategy for Europe's Electricity Networks of the Future. EUROPEAN COMMISSION, Luxembourg, 2006.

[4] Robin Podmore, Fellow-IEEE, and Marck Ramon Robinson. The Role of Simulators for Smart Grid Development. IEEE Transactions On Smart Grid. September 2010.

[5] Mathavi Ms. S, Vanitha Mrs. D, Jeyanthi Mrs. S, Senthil Mr. P. kumaran. The Smart Home: Renewable Energy Management System for Smart Grid Based On ISM Band Communications. International Journal of Scientific \& Engineering Research. March 2012.

[6] Deepak P, Bibhudatta S, Sahoo BPS. Effective Machine to Machine Communications in Smart Grid Networks. ARPN Journal of Systems and Software. January 2012; 18-22.

[7] Oleg Gulich, M.Sc. (Tech.) Ville Karttunen, Prof. JarmoPartanen. TECHNOLOGICAL AND BUSINESS CHALLENGES OF SMART GRIDS. Master Thesis, 2010.

[8] Erol-Kantarci M, Mouftah HT. Supply and load management for the smart distribution grid using wireless networks. Electronics, Communications and Computers (JEC-ECC), 2012 Japan-Egypt Conference. March 2012; 145,150, 6-9.

[9] Erol-Kantarci M, Mouftah HT. Wireless Sensor Networks for domestic energy management in smart grids. Communications (QBSC), 2010 25th Biennial Symposium on. 12-14 May 2010; 63-66.

[10] Erol-Kantarci M, Mouftah HT. Using Wireless Sensor Networks for Energy-Aware Homes in Smart Grids. Computers and Communications (ISCC), 2010 IEEE Symposium on. 22-25 June 2010; 456-458.

[11] Korkua SK, Thinsurat K. Design of ZigBee based WSN for Smart Demand Responsive Home Energy Management System. Communications and Information Technologies (ISCIT), 2013 13th International Symposium on. 4-6 Sept. 2013; 549-554

[12] Devidas AR, Subeesh TS, Ramesh MV. Design and Implementation of User Interactive Wireless Smart Home Energy Management System. Advances in Computing, Communications and Informatics (ICACCI), 2013 International Conference on. 22-25 August 2013; 626-631.

[13] Maghsoodi NH, Haghnegahdar M, Jahangir AH, Sanaei E. Performance Evaluation of Energy Management System in Smart Home using Wireless Sensor Network. Smart Grids (ICSG), 2012 2nd Iranian Conference on. 24-25 May 2012; 1-8.

[14] Mahmood A, Khan I, Razzaq S, Najam Z, Khan NA, Rehman MA, Javaid N. Home Appliances Coordination Scheme for Energy Management (HACS4EM) using Wireless Sensor Networks in Smart Grids. 5th International Conference on Ambient Systems, Networks and Technologies (ANT-2014), Procedia Computer Science. 2014; 469-476.

[15] Prasath KB, Vijayakumar S, Prasath S. Kumar. An Efficient Approach for Home Energy Management System. International Journal of Engineering Science Inventio. December 2013; 49-58.

[16] Erol-Kantarci M, Mouftah HT. TOU-Aware Energy Management and Wireless Sensor Networks for Reducing Peak Load in Smart Grids. Vehicular Technology Conference Fall (VTC 2010-Fall), 2010 IEEE 72nd. 6-9 Sept. 2010; 1-5.

[17] Baig1 F, Mahmood A, Javaid N, Razzaq S, Khan N, Saleem Z. Smart Home Energy Management System for Monitoring and Scheduling of Home Appliances Using Zigbee. J. Basic. Appl. Sci. 2013; 880-891.

[18] Erol-Kantarci M, Mouftah HT. Wireless Sensor Networks for Smart Grid Application. IEEE 24-26 April 2011; 1-6.

[19] Khan I, Mahmood A, Javaid N, Razzaq S, Khan RD, IlahiM. Home Energy Management Systems in Future Smart Grids. Nadeem javaid, COMSATS Institute of IT, Islamabad, www.njavaid.com. 2013; 459-464. 
[20] Javaid N, Khan I, Ullah MN, Mahmood A, Farooq MU. A Survey of Home Energy Management Systems in Future Smart Grid Communications. Broadband and Wireless Computing, Communication and Applications (BWCCA'13), 2013 Eighth International Conference on. 28-30 October 2013; 459,464.

[21] Erol-Kantarci, M.; Mouftah, H.T., "Energy-Efficient Information and Communication Infrastructures in the Smart Grid: A Survey on Interactions and Open Issues," in Communications Surveys \& Tutorials, IEEE , vol.17, no.1, pp.179-197, Firstquarter 2015.

\section{Authors}

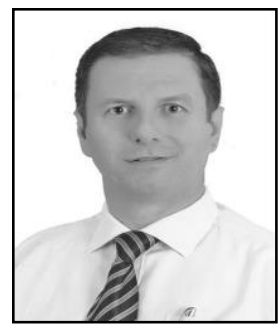

Wail Mardini is an Associate professor of Computer Science at Jordan University of Science and Technology (JUST)/ Jordan since 2006. Dr. Mardini received his Masters and Ph.D. degree in Computer Science from University of New Brunswick/Canada at 2001 and University of Ottawa/Canada at 2006, respectively. Dr. Mardini research interests include Wireless Mesh Networks, Wireless Sensor Networks, Optical Network Survivability, WiMax Technology, Scheduling in Parallel Computing, and Intrusion Detection in database Techniques.

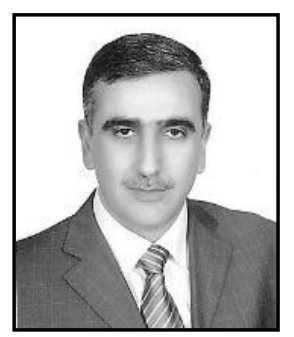

Firas A. ALbalas is an Assistant Professor in the Department of Computer Science, Jordan University of Science and Technology, Irbid, Jordan. He received his $\mathrm{PhD}$ in Computer Science from Glamorgan (South Wales) University, Cardiff, UK in 2009. His current research interests include mobile computing, ad hoc networks and sensor networks.

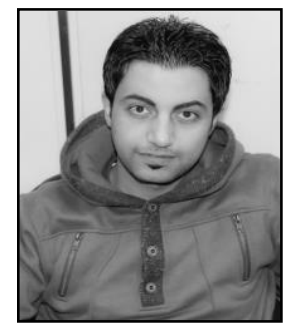

Qusai Obeidat is currently a Programmer and System Analyst at Electricity Distribution Company (EDCO) in Amman/Jordan. Obeidat worked before as a teacher assistant at the Computer Science department at Jordan University of Science and Technology (JUST) /Jordan. He received his Master's degree in Computer Science from JUST in 2015 and his Bachelor degree in Computer Information Systems from JUST in 2011. Obeidat research interests include Wireless Sensor Networks, Web technologies, and mobile-based application development.

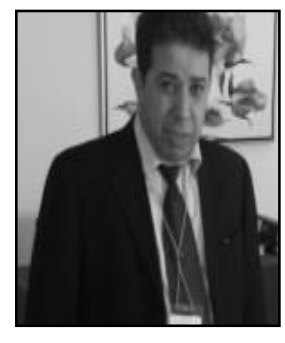

Muneer Masadeh Bani Yassein received his $\mathrm{PhD}$ degrees in Computer Science from the University of Glasgow, U.K, He is currently an associate professor in the Department of Computer science at Jordan University of Science and Technology (JUST). Dr. Bani Yassein served as Head of Department of Computer Science from 2008 to 2010, Vice Dean of the Faculty of Computer and Information Technology from 2010 to 2012, and from 2013-2014. Dr. Bani Yassein is currently conducting research in Mobile Ad hoc Networks, Wireless sensors Networks, Cloud Computing, Embedded Systems, Computational Mathematics, Internet of Things. Dr. Bani Yassein, published over 80 technical papers in international journals and conferences. Dr. Bani Yassein is member of IEEE and he is a member of the technical programs of several journals and conferences. 
International Journal of Smart Home

Vol. 11, No. 5 (2017) 